\title{
tRNA-guanine transglycosylase from Escherichia coli: Recognition of dimeric, unmodified tRNATyr
}

\author{
AW Curnow, GA Garcia* \\ Interdepartmental Program in Medicinal Chemistry. College of Pharmacy. University of Michigan, \\ Ann Arbor, Michigan 48109-1065, USA
}

(Received 5 May 1994; accepted 20 September 1994)

\begin{abstract}
Summary - In order to probe the interaction between tRNA and the tRNA hypernodifying enzyme, tRNA-guanine transglycosylase (TGT) from Escherichia coli, we have undertaken the generation of $E$ coli tRNATyr and analogues. During efforts to adapt currently available in vitro transcription techniques we encountered difficulties attributable to dimerization of the tRNA products. $E$ coli tRNATyr has previously been characterized for its ability to form a dimer in solutions of suitable salt concentrations at appropriate temperatures (Yang SK, Söll DG. Crothers DM (1972) Biochemistry 11, 2311-2320; Rordorff BF, Kearns DR (1976) Biochemistry 15, 3320-3330). We have appliec: similar techniques to our unmodified analogue of $E$ coli tRNATyr and produced both monomeric and dimeric forms of $E$ coli tRNATyr. In this report we find that the ciiner does serve as a substrate for modification by TGT. While both the conformers are equal in terms of $V_{\text {max }}$ (within experimental error) a 2.5 -fold increase in $K_{M}$ occurs when going from monomer to dimer. This suggests that TGT prefcrentially binds the monomer but once either conformer is bound will catalyze the modification reaction equally well. We have also compared the results for the two conformers to our previous data of an RNA minihelix corresponding to the anticodon arm of $E$ coli tRNATyr. Here we find that our earlier conclusion, that the recognition elements for TGT are localized within the anticodon arm of cognate IRNAs, is supported.
\end{abstract}

queuine / transglycosylase / tRNA / modified nucleotide / band shift assay

\section{Introduction}

Molecular recognition is a critically important process which is necessary for enzymes to distinguish substrate molecules from structurally similar molecules. The tRNAs and the various enzymes with which they interact provide particularly good examples of this phenomenon. In these cases recognition, and more importantly discrimination between cognate and noncognate tRNAs, can be quite difficult due to the large degree of conserved sequence [1] and the common tertiary structure [2] in tRNAs.

One enzyme which encounters this situation is tRNA-guanine transglycosylase (TGT, EC 2.4.2.29) [3]. In Escherichia coli, this enzyme catalyzes the replacement of the anticodon guanine (guanine 34 ) in

\footnotetext{
*Correspondence and reprints

Abbreviations: HEPES, hydroxyethylpiperazine-ethylsulfonate; DTT, dithiothreitol; EDTA, ethylenediaminetetraacetic acid; NTP, ribonucleotide triphosphate; ECY2M, E coli tRNATyr monomeric form; ECY2D, $E$ coli tRNATyr dimeric form; TGT, tRNA-guanine transglycosylase.
}

tRNAs Tyr, Asp, Asn, and His with 7-aminomethyl-7deazaguanine, pre $Q_{1}$. Ultimately, $p_{r e} Q_{1}$ is converted to the hypermodified base queuine. In order to investigate the nature of this modification we undertook the synthesis of an unmodified analogue of $E$ coli tRNATyr (fig 1) via the in vitro transcription method suggested by Milligan and Uhlenbeck [4]. As a consequence of conditions during transcription and purification, two distinct tRNA conformers were obtained. These conformers appeared to correspond to monomeric and dimeric tRNA.

Dimerization has been demonstrated in heterogeneous RNA mixtures $[5,6]$ and characterized for specific tRNAs including: yeast tRNA ${ }^{\text {Ser }}$ [7], tRNAGly [8] and tRNA $A$ Ia [9], and the most relevant to this work, $E$ coli tRNATyr [10]. While various groups have studied the effects of aggregate formation in several enzyme systems the biological significance of such interactions has not yet been fully established.

Loehr and Keller [9] found that alanyl-tRNA synthetase was able to correctly charge dimers of yeast tRNAAla with alanine and proposed a structure for a possible tRNAAla dimer conformation. Investigations with yeast tRNA ${ }^{\text {Ser }}$ produced analogous results in that 


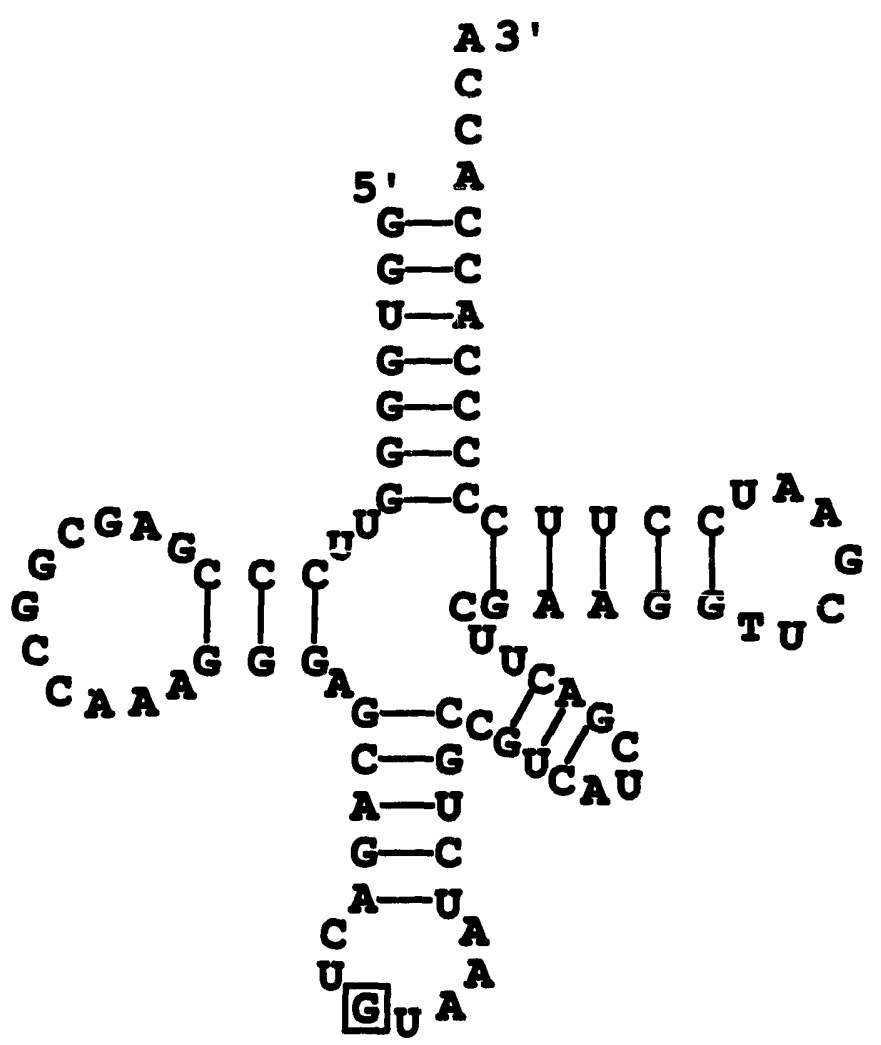

Fig 1. The primary sequence and secondary structure of $E$ coli tRNA tyr. Guanosine-34 is highlighted.

aggregation of the tRNA species did not seem to inhibit the aminoacylation by the cognate aminoacyltRNA synthetase [7]. The researchers surmised, in both instances, that the motifs related to recognition were maintained in the aggregate forms. On the contrary, Yang et al [10] found that, once formed, a dimer of $E$ coli tRNATyr was no longer capable of aminoacylation by tyrosyl-tRNA synthetase. In addition, when the dimer was heated in an appropriate buffer system the monomer tRNA could be reformed and aminoacylation proceeded. Finally, a structure for the tRNATyr dimer was proposed.

Subsequently, a proton NMR study of the base pairing in the $E$ coli tRNATyr dimer and monomer led to a solution structure model for the dimer being proposed (fig 2) [11]. In this structure an extended, hybrid double helix is formed from the acceptor stems and TYC arms of each individual tRNA molecule while the anticodon arms of each monomer remain intact.

In previous work [12] we established that an RNA minihelix analogous to the anticodon arm of $E$ coli tRNATyr acts as a substrate for modification by TGT. Since the proposed dimer maintains the structure of the anticodon arms we endeavored to investigate the nature of the interaction between TGT and an unmodified dimer of $E$ coli tRNATyr.

\section{Materials and methods}

\section{Reagents}

Chemical reagents were purchased from Sigma, Boehringer Mannheim, and Gibco BRL. Nucleoside triphosphates and Rnasin were from Boehringer Mannheim. Bst NI restriction endonuclease was from Stratagene. $8-\left[{ }^{14} \mathrm{C}\right]$ Guanine was from Moravel. Biochemitals. TGT was isolated from an overexpressing clone as described previously [13]. T7 RNA polymerase was isolated from $E$ coli BL21/pARI219 following the procedure of Grodberg and Dunn [14].

\section{Preparation and purification of the IRNA transcript}

Unmodified $E$ col: tRNATyr was produced via the transcription of double-stranded DNA template in an in vitro system as previously described [12].

\section{Formation of ECY2 conformers}

Monomerization and dimerization is based on the work of Yang $e t$ al [10]. The monomer of $E$ coli tRNATyr (ECY2M) was formed in $5 \mathrm{mM}$ HEPES (pH 7.5) and $400 \mu \mathrm{M} \mathrm{MgCl}$ with the concentration of tRNA varying from 20 to $40 \mu \mathrm{M}$. Monomerization proceeds by incubating the tRNA at $68^{\circ} \mathrm{C}$ for $1 \mathrm{~h}$ and then cooling quickly to $4^{\circ} \mathrm{C}$ and maintaining at $4^{\circ} \mathrm{C}$ for $1 \mathrm{~h}$. The dimer (ECY2D) is formed in a buffer containing $5 \mathrm{mM}$ HEPES ( $\mathrm{pH} \mathrm{7.5)} \mathrm{and} 0.5$ to $1 \mathrm{M} \mathrm{NaCl}$ with the concentration of tRNA in an optimal range of 50 to $100 \mu \mathrm{M}$. Dimerization occurs when the tRNA is incubated at $50^{\circ} \mathrm{C}$ for $4 \mathrm{~h}$ and then cooled quickly to $4^{\circ} \mathrm{C}$ and maintained for $1 \mathrm{~h}$.

\section{Gel filtration chromatography}

The chromatography was carried out on a Pharmacia FPLC system using a Superose 12 column (HR 10/30, Pharmacia). The samples were injected onto the column, and eluted isocratically in $10 \mathrm{mM}$ HEPES (pH 7.5), I mM EDTA, and $250 \mathrm{mM} \mathrm{NaCl}$. The peak fractions were collected and subjected to polyacrylamide gel electrophoresis.

\section{Polyacrylamide gel electrophoresis}

All electrophoretic analyses were performed on a PhastSystem (Pharmacia) following PhastSystem protocols. Native protein gels were run by applying the samples and standards directly on an 8 to $25 \%$ gradient gels (Pharmacia PhastGel) and were electrophoresed using PhastGel native buffer strips $(880 \mathrm{mM}$ L-alanine, $250 \mathrm{mM}$ Tris (pH 8.8), 2\% agarose). For native tRNA gels, the samples were directly applied to $20 \%$ homogeneous gels (Pharmacia PhastGel) and electrophoresed using native buffer strips. In the case of tRNA denaturing gels, high density gels (Pharmacia PhastGel) were prepared by saturating with TBE/urea biffer $(8.9 \mathrm{mM}$ Tris base, $8.9 \mathrm{mM}$ boric acid, $0.2 \mathrm{mM}$ EDTA, $7 \mathrm{M}$ urea (pH 8.0) and the samples were prepared by mixing the tRNA 1:1 with RNA loading buffer (10 M urea, $5 \mathrm{mM}$ Tris pH 8.0), $5 \mathrm{mM}$ boric acid, $1 \mathrm{mM}$ EDTA, $0.01 \%$ xylene cyanole, $0.01 \%$ bromophenol blue) and 
heating at $70^{\circ} \mathrm{C}$ for $10 \mathrm{~min}$. The samples were then applied to the gel and electrophoresed using TBE buffer strips $(8.9 \mathrm{mM}$ Tris base, $8.9 \mathrm{mM}$ boric acid, $0.2 \mathrm{mM}$ EDTA, 2\% agarose). For the gel shown in figure 6, ca $5 \mu \mathrm{g}$ of protein $(4 \mu$ of incubation mixture) was loaded in each lane. TGT $(15 \mu \mathrm{M})$ was incubated for $15 \mathrm{~min}$ at $37^{\circ} \mathrm{C}$ in $100 \mathrm{mM}$ HEPES (pH 7.5), alone (lane 2) and + $10 \mu \mathrm{M}$ tRNA ECY2 (lane 4). Both of the samples were divided into two equals volumes and ribonuclease $A(50 \mu \mathrm{g} / \mathrm{ml}$ final concentration) was added to one sample of each (lanes 3 , 5). All four samples were incubated for an additional $15 \mathrm{~min}$ at $37^{\circ} \mathrm{C}$. Upon completion of electrophoresis, protein gels were stained with Coomassie blue and tRNA gels were stained with ethidium bromide and visualized on a UV transilluminator.

\section{Kinetic assays}

The kinetic parameter determinations were performed in a buffer consisting of $100 \mathrm{mM}$ HEPES at pH 7.5,10 mM MgCl , and $100 \mathrm{mM}$ DTT. The concentration of $[8-14 \mathrm{C}]$-guanine was maintained at $10 \mu \mathrm{M}$ while the concentration of the tRNA conformers was varied from $0.5 \mu \mathrm{M}$ to $20 \mu \mathrm{M}$. The general procedure included incubating a $400 \mu \mathrm{l}$ mixture of buffer, substrate, and $12.6 \mu_{\mathrm{g}} \mathrm{ml}$ TGT at $37^{\circ} \mathrm{C}$. At 2 min intervals $75 \mu \mathrm{l}$ aliquots were removed from the reaction mixture. The aliquots were quenched in 5\% TCA and filtered onto glass fiber filters (Whatman, GF/C). The filters were then washed three times with 5\% TCA and once with ethanol, dried, and quantitated via
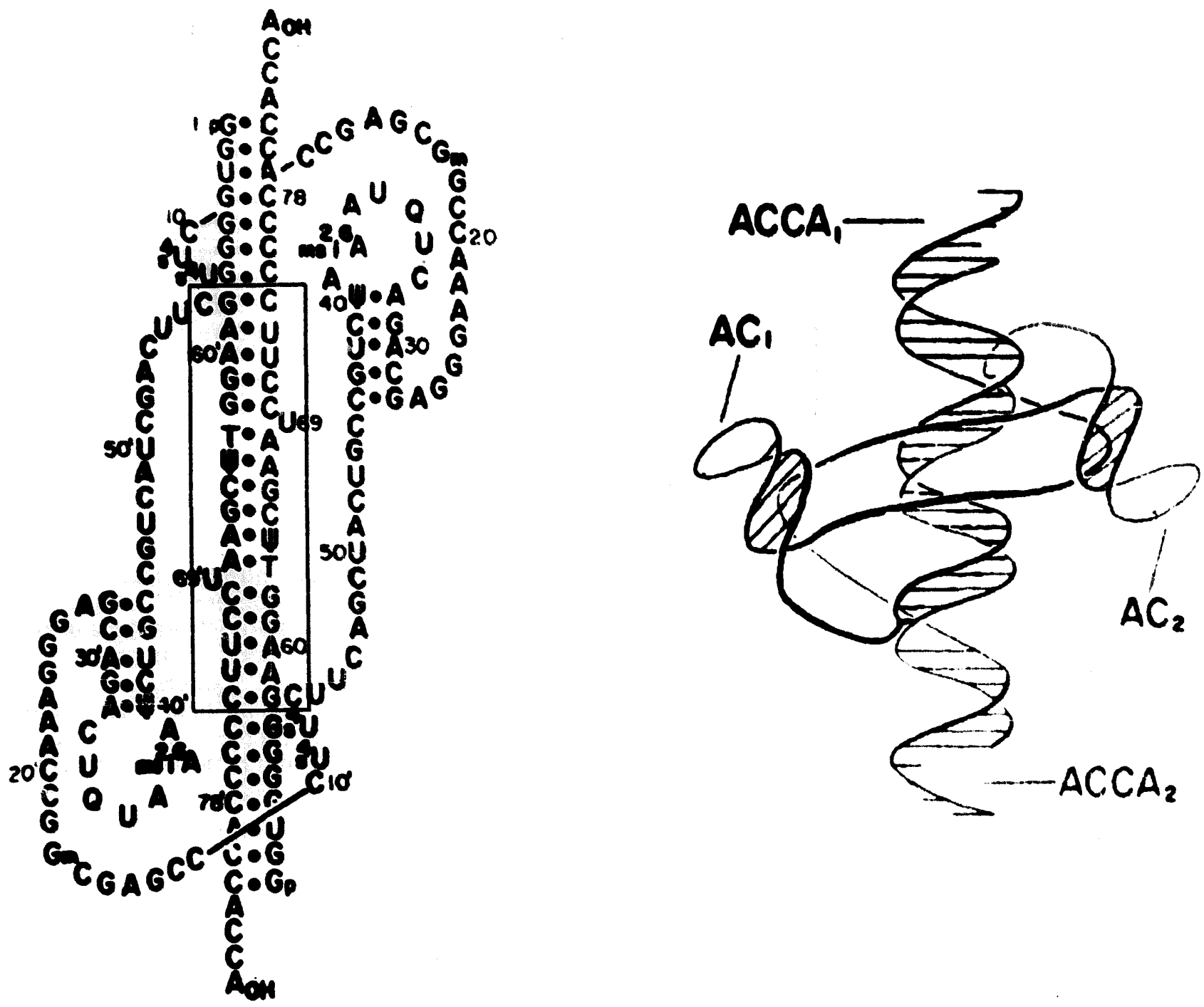

Fig 2. Secondary (left) and tertiary (right) structure models of the dimer of $E$ coli tRNATyr proposed by Rordorf and Kearns [11]. The intermolecular base pairs in the secondary structure model are enclosed in a box (adapted with permission from Rordorff and Kearns, Biochemistry 15, 3320-3330. Copyright (1976) American Chemical Society). 

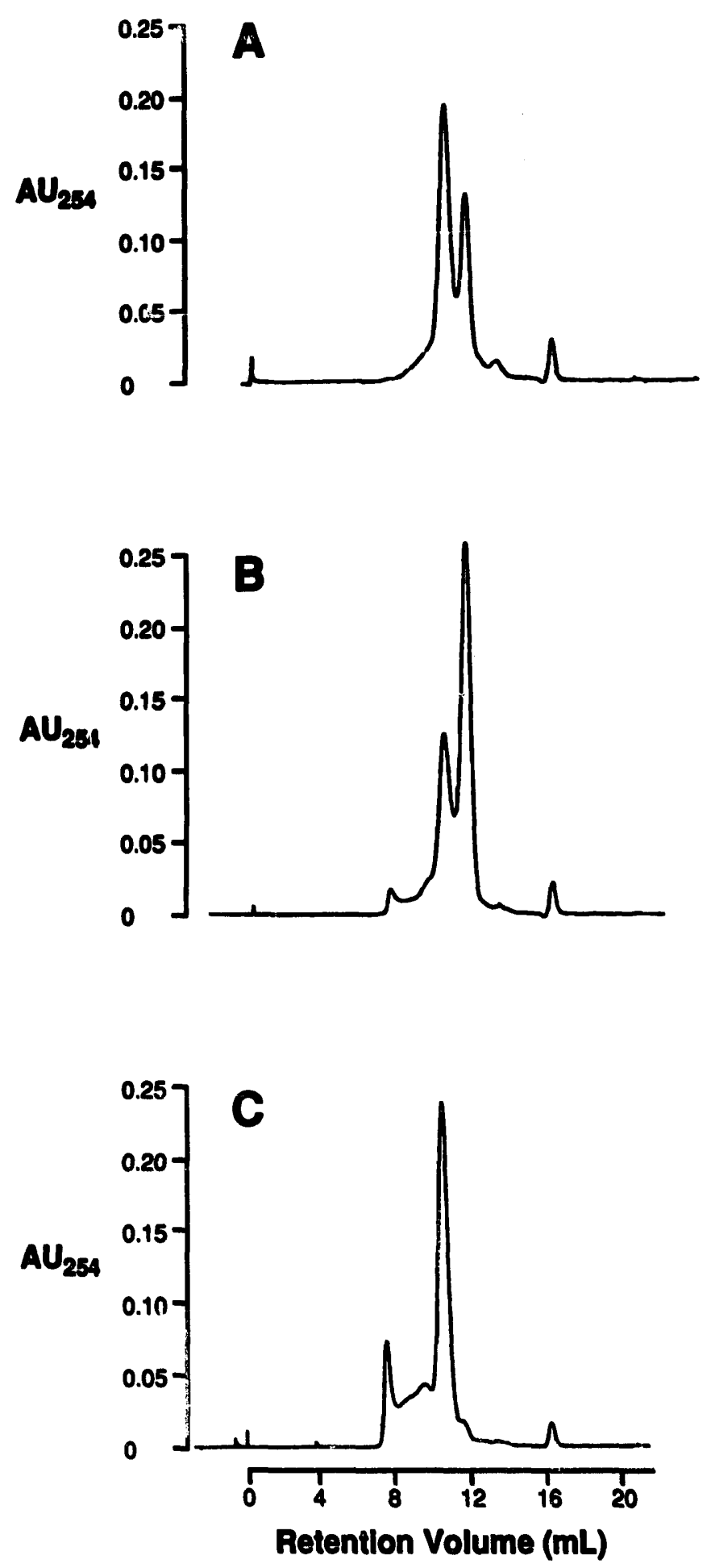

Fig 3. Superose 12 gel filtration separation of ECY2M and ECY2D. A. Untreated $E$ coli tRNATyr. B. Transcript after heating at $68^{\circ} \mathrm{C}$ for $1 \mathrm{~h}$ in $400 \mu \mathrm{M} \mathrm{MgCl}$. C. Transcript after heating at $50^{\circ} \mathrm{C}$ for $4 \mathrm{~h}$ in $1 \mathrm{M} \mathrm{NaCl}$ is described in Materia's and methods. liquid scintillation counting. Initial velocities were determined by linear regression and from these data Michaelis-Menten parameters were determined by non-linear regression.

\section{Results}

Formation and characterization of E coli tRNATrr conformers

We have modified published procedures so that both dimeric and monomeric $E$ coli tRNATyr can be produced in a buffer system compatible with our TGT assay. Gel filtration chromatography was used to characterize the conformers. Figure 3 shows the results of the separation: (A), the untreated $E$ coli tRNATyr transcription product resuspended in gel filtration buffer; (B), sample from $A$ heated at $68^{\circ} \mathrm{C}$ in the presence of $400 \mu \mathrm{M} \mathrm{MgCl}$; and (C) sample from A heated at $50^{\circ} \mathrm{C}$ in the presence of $1 \mathrm{M} \mathrm{NaCl}$. The incubation at $68^{\circ} \mathrm{C}$ furces tite IRNA to assume the monomer conformation, while lower temperature and high salt concentration generate the dimer. At identical points in both chromatograms, prior to the major peak, a small peak elutes. This peak is presumably due to some contaminating, high molecular mass species generated during the prolonged exposure to high salt and elevated temperatures.

The conformers were also separated via polyacrylamide gel electrophoresis (fig 4). Under native conditions (fig 4A) two bands are clearly distinguishable that correspond to ECY2M and ECY2D (lane 1). Lanes 2 and 3 represent the monomerization procedure, laine $\mathbf{2}$ is the sample prior to gel filtration and lane 3 is the predominant peak from figure 3B which agrees with ECY2M. The dimerization product is represented by lanes 4 and 5 . Here lane 4 is the dimerized sample before gel filtration and lane 5 is the major peak, ECY2D, from figure 3C.

Figure 4B shows the results from denaturing polyacrylamide gel electrophoresis. In lane 1 is the untreated $E$ coli tRNATyi transcript, a mixture of ECY2M and ECY2D and in lane 3 is a sample of ECY2D. After being exposed to $7 \mathrm{M}$ urea both samples denature and migrate to positions equivalent to an ECY2M sample which has been similarly treated (lane 2).

\section{IRNA.TGT interaction}

The interaction of ECY2M and ECY2D with TGT in the absence of guanine was investigated by native PAGE (fig 5). Lane 2 shows the protein alone migrating to an apparent nolecular mass of $127 \mathrm{kDa}$. This represents the protein in its native trimeric state. The enzyme incubated in the presence of ECY2M appears in lane 3. In this instance the protein dissociates into two bands, $69 \mathrm{kDa}$, the ECY2M.TGT (monomer) complex, and $150 \mathrm{kDa}$, the ECY2M.TGT 


\section{A}

\section{5}

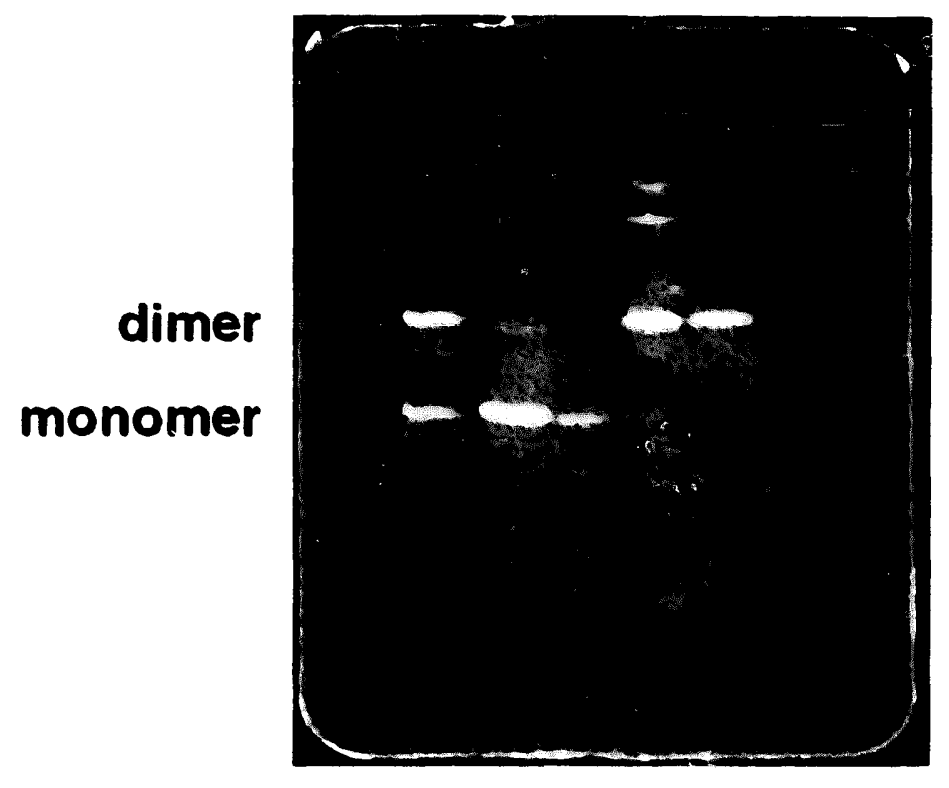

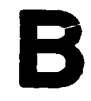

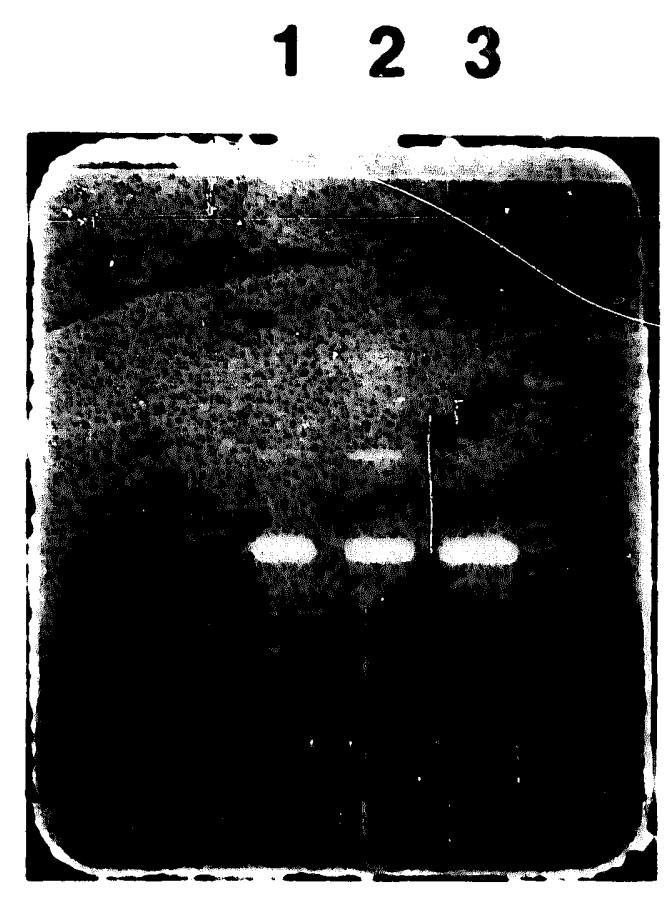

Fig 4. Native-PAGE of ECY2 and ECY2D and denaturing-PAGE of ECY2 and ECY2D. A. Native-PAGE of ECY2 and ECY2D. Lane 1, untreated $E$ coli IRNATyr (prior to separation in figure 3A); lane 2, ECY2 (prior to separation in figure 3B); lane 3, major peak from figure 3B; lane 4, ECY2D (prior to separation in figure 3C); lane 5, major peak from figure 3C. B. Denaturing-PAGE of ECY2 and ECY2D. Lane 1, untreated $E$ coli tRNATyr (prior to separation in figure 3A); lane 2, ECY2 (prior to separation in figure 3B); lane 3, ECY2D (prior to separation in figure 3C).

(trimer) complex. In lane 4 the enzyme is incubated with ECY2D and again two bands appear. Here the bands correspond to $96 \mathrm{kDa}$, the ECY2D.TGT (monomer) complex, and $180 \mathrm{kDa}$, the ECY2D.TGT (trimer) complex.

In order to further investigate the band shift phenomenon, we incubated ECY2M.TGT complex with ribonuclease. In figure 6 the TGT trimer and ECY2M. TGT complex bands appear (lanes 3,4 ). When the ECY2M-TGT complex sample is treated with ribonuclease three bands appear corresponding to the TGT trimer, a band that migrates at a slightly higher $M_{\mathrm{r}}$ and a fainter band at a lower $M_{\mathrm{r}}$ than the ECY2M.TGT complex (lane 5). The lower band may be due to a TGT complex with partially digested ECY2M. The band at the slightly higher $M_{\mathrm{r}}$ than the TGT trimer may be due to a TGT trimer complex with partially digested ECY2M.

\section{in vitro kinetics}

The kinetic studies were performed in the presence of $10 \mu \mathrm{M} 8-\left[{ }^{14} \mathrm{C}\right]$ guanine. This was selected due to pre- 
vious experiments which established a guanine $K_{M}=$ $0.98 \mu \mathrm{M}$ [12]. Plots of initial velocity versus RNA concentration (fig 7) demonstrate that MichaelisMenten kinetics are followed for each conformer and

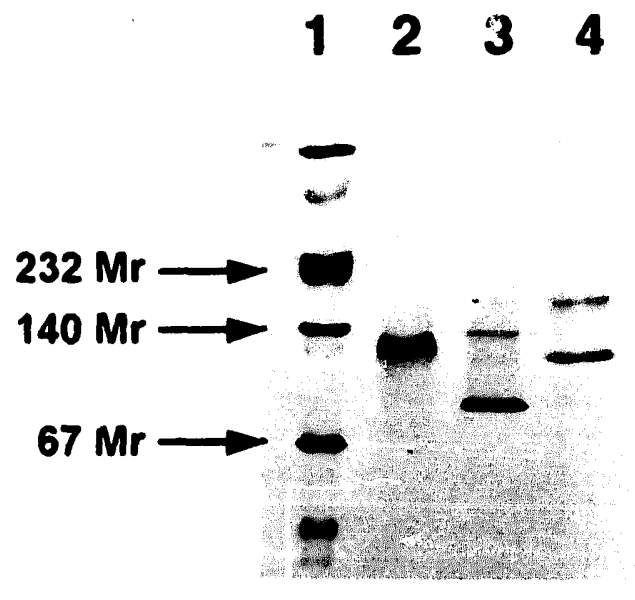

Fig 5. Native-PAGE of ECY2Mí and ECY2D complexes with TGT. Lane 1, $M_{\text {r }}$ standards; lane 2, $5 \mu \mathrm{M}$ TGT without substrates; lane 3, $5 \mu \mathrm{M}$ TGT + $15 \mu \mathrm{M}$ ECY2; lane 4, $5 \mu \mathrm{M}$ TGT + 15 بM ECY2D. The enzyme was incubated at $37^{\circ} \mathrm{C}$ for 10 inin in $100 \mathrm{mM}$ HEPFS, pH 7.5 with the indicated concentration of substrate. The gel was then run at a constant temperature of $5^{\circ} \mathrm{C}$.

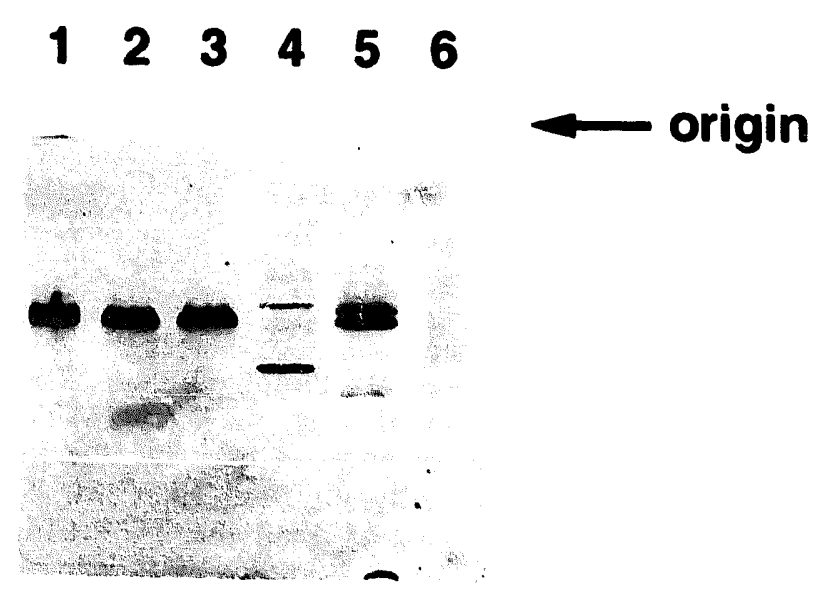

Fig 6. Native-PACE of TGT and TGT.tRNA complex. Approximately equal amounts of TG'T protein were applied to each lane. Lane 1, TGT standard; lane 2, TGT control; lane 3, TGT + ribonuclease; lane 4, TGT + ECY2M; lane 5, TGT + ECY2M + ribonuclease; lane 6, ribonuclease.
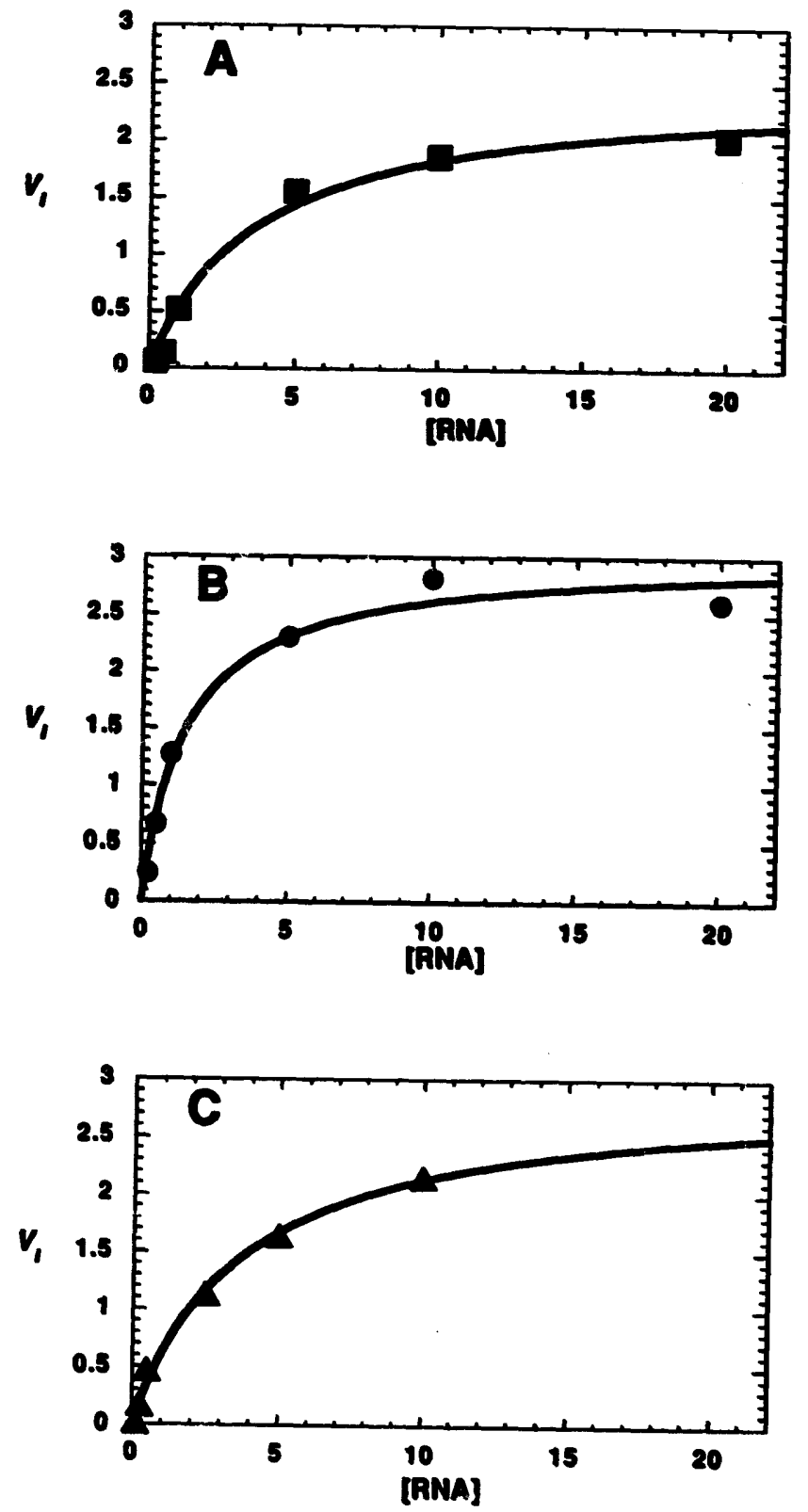

Fig 7. Michaelis-Menten plots of untreated $E$ coli tRNATy,, ECY2M, and ECY2D. A. Untreated E coli TRNATyr. B. ECY2M. C. ECY2D. Solid curves are calculated fits of the data by non-linear regression. $V_{i}$ units are $\mu \mathrm{M} \mathrm{s}^{-1} \mathrm{mg}^{-1}$ and (RNA) units are in $\mu \mathrm{M}$.

a mixture of both conformers. Non-linear regression analysis was used to fit the initial velocity data and are displayed as the solid curves in figure 7. The kinetic parameters $\left(K_{\mathrm{M}}, V_{\max }\right.$, and $\left.V_{\max } / K_{\mathrm{M}}\right)$ for $\mathrm{ECY} 2$, ECY2D, and a mixture of the two from the above fits are shown in table I along with the standard errors of the fits. 


\section{Discussion}

Using the in vitro generation system suggested by Milligan and Uhlenbeck [4], we successfully implemented a system for the in vitro generation and purification of $E$ coli $t R N A T y r$ analogues to investigate the molecular processes involved in substrate recognition by $E$ coli TGT. One problem related to the system was the dimerization of the transcripts during the purification process. Dimerization of tRNA is not rare albeit most reports of the phenomenon are associated with the isolation and recovery of in vivo generated tRNA and predate in vitro generated tRNA studies. Furthermore, the biological significance of tRNA dimerization remains unresolved. To further our investigation into the elements required for recognition and modification of tRNA and to expand the knowledge base regarding the biological significance of tRNA aggregation we embarked on studies to produce both a monomeric and dimeric $E$ coli tRNATyr (ECY2M and ECY2D respectively) in a buffer system conductive to characterization of the interaction of each species with TGT.

As demonstrated in figures 3 and 4 we have successfully generated tRNATyr monomer (ECY2M) and dimer (ECY2D) in the appropriate buffer system. The gel filtration and native-PAGE experiments show that the transcription and purification process provides two major products which display migration patterns consistent with the monomer and dimer conformations (fig 3A, fig 4, lane 1). Upon heating at appropriate temperatures in the presence of different cations the transcript conformation can be driven into either the monomeric (fig 3B, fig 4A, lanes 2 and 3 ) or dimeric (fig 3C, fig 4A, lanes 4 and 5) conformations. An additional peak, presumably due to higher order aggregates of tRNA, appears in the treated samples. The fact that the peak is larger in the ECY2D sample than in the ECY2M sample suggests that the for- mation of higher order aggregates is dependent upon the length of time the nucleic acid is exposed to elevated temperatures, the concentration at which the nucleic acid is treated, and/or the valency of the cation in the treatment buffer. The higher order aggregates also appear in the native-PAGE with a more intense band in the ECY2D lane (fig 4A, lane 4). The most compelling evidence for dimer and monomer formation is provided by the denaturing-PAGE. After the tRNA is denatured by exposure to $7 \mathrm{M}$ urea at $70^{\circ} \mathrm{C}$ for $10 \mathrm{~min}$ (see Materials and methods) each of the three samples exhibits a single band which migrates to a point corresponding to monomeric ECY2.

We have previously reported that $E$ coli TGT purified from our overexpressing clone appears to be oligomeric [13]. Native-PAGE of TGT prepared from an optimized protocol shows only a single band corresponding to an apparent trimeric form of TGT which has been confirmed by chemical cross-linking and denaturing PAGE (Garcia and Chong, unpublished). When incubated in the presence of saturating amounts of tRNA, the TGT band shifts to a position consistent with the formation of a tRNA.TGT monomer complex (fig 6, lane 4). Ethidium bromide staining of the native-PAGE indicates that tRNA is present in the TGT.tRNA complex band (data not shown). This trimer to monomer dissociation appears to be reversible as after treatment with ribonuclease, the TGT.tRNA complex migrates predominantly as the TGT trimer (fig 6, lane 5). The exact significance of the TGT trimer to monomer dissociation upon binding tRNA is unknown.

It has been suggested that tRNA aggregation could act as a mechanism for the control of tRNA function. For instance dimerization of $E$ coli tRNATyr has been found to lead to the loss of the aminoacylation activity by tyrosyl-tRNA synthetase [10]. Studies to elucidate which recognition elements found in B stearothermophilus tRNA ${ }^{\text {Tyr }}$ allow for aminoacylation by tyrosyl-

Table I. Kinetic parameters for TGT substrates. Standard errors are shown in parentheses. The concentrations of the untreated FCY2 transcript were calculated assuming the presence of monomer only. The values for ECY2-A1 have been recalculated from the data in Curnow et al [12] to account for the difference in the amount of enzyme used and to make the units consistent. The $\mathrm{V}_{\text {max }} / K_{\mathrm{M}}$ ratio was calculated relative to $\mathrm{ECY} 2 \mathrm{M}$.

\begin{tabular}{lcccc}
\hline Conformer & $\begin{array}{c}K_{m} \\
(\mu M)\end{array}$ & $\begin{array}{c}V_{\max } \\
\left(\mu M \cdot s^{-1} \cdot m g^{-1}\right)\end{array}$ & $\begin{array}{c}V_{\operatorname{mal}} / K_{m} \\
\left(s^{-1} \cdot m g^{-1}\right)\end{array}$ & $\begin{array}{c}V_{\max } / K_{m} \\
\text { ratio }\end{array}$ \\
\hline $\begin{array}{l}\text { Untreated } \\
\text { ECY2 transcript }\end{array}$ & $3.7(0.9)$ & $2.5(0.19)$ & $0.68(0.02)$ & $1: 2.8$ \\
Monomer (ECY2M) & $1.6(0.3)$ & $3.0(0.17)$ & $1.95(0.02)$ & $1: 1$ \\
Dimer (ECY2M) & $3.8(0.7)$ & $2.9(0.23)$ & $0.77(0.02)$ & $1: 2.5$ \\
Stem-loop (ECY2-A1) & $9.6(0.7)$ & $0.39(0.01)$ & $0.04(0.01)$ & $1: 50$ \\
\hline
\end{tabular}


tRNA synthetase have shown that adenine- 73 acts as a discriminator base for tyrosyl-tRNA synthetase recognition but only in conjunction with the overall structure of $B$ stearothermophilus tRNATyr [15]. If a similar gross structural requirement exists for the $E$ coli tyrosyl-tRNA synthetase, then formation of the dimer and concomitant loss of native structure could result in loss of aminoacylation activity.

Other IRNA species, such as yeast IRNASer and yeast tRNAAla, retain the ability to be aminoacylated by their cognate aminoacyl-tRNA syithetase upon dimer formation [7, 9]. Loehr and Keller [9] demonstrated that, once formed, dimers of yeast tRNAAlit were correctly charged with alanine by alanyl-tRNA synthetase. They went on to propose a structure for the tRNA Ala dimer conformation. In this structure two G3-U70 discriminator base pairs and two acceptor stems are formed as hybrids of the two individual tRNA molecules. In independent work, alanyl-tRNA synthetase has displayed aminoacylation activity with RNA duplexes comprising the CCA acceptor, the G3-U70 discriminator base pair, and as few as four base pairs of $E$ coli tRNAAla acceptor stem [16], thus demonstrating that the only structural requirement for aminoacylation is the acceptor stem itself. These investigations illustrate that when key structural recognition elements are maintained in grossly varied structures enzyme recognition and catalysis can be preserved.

We have previously shown that ECY2-A1, a minihelix analogue of $E$ coli tRNATyr, corresponding to the anticodon stem and loop, had a $K_{\mathrm{M}}$ of $9.6 \mu \mathrm{M}$ and a $V_{\max }$ of $0.39 \mu \mathrm{M} \cdot \mathrm{s}^{-1} \cdot \mathrm{mg}^{-1}$ with TGT, indicating that up to $80 \%$ of the tRNA sequence can be removed resulting in only a 20 -fold decrease in substrate specificity [12]. This 20 -fold difference could be attributed to other specific and/or non-specific interactions between TGT and the full length tRNA which do not occur with the minihelix. Considering the satisfactory activity of TGT with ECY2-A1 and the published structure of dimerized tRNATyr, with the key TGT structural recognition element intact, ie the anticodon stem and loop motifs, we felt that ECY2D was very likely to be a substrate for TGT modification.

In table I we present the results of the kinetic parameter determinations. We find that in cases where the full length tRNA is involved the $V_{\max }$ is equal within experimental error. However, both the dimer and the untreated $E$ coli tRNATyr have $K_{\mathrm{M}}$ 's which are elevated approximately 2.5 -fold over the $K_{\mathrm{M}}$ for the monomer resulting in a $V_{\text {max }} / K_{\mathrm{M}}$ which is decreased 2.5 -fold accordingly. This suggests that the enzyme preferentially binds the monomer over dimer, but once bound either conformation undergoes catalysis equivalently.
In our earlier study we found that ECY2-A1 had a 20 -fold lower specificity, reflected in $V_{\max } / K_{\mathrm{M}}$, than full-length, unmodified $E$ coli tRNA ${ }^{\text {Tyr. Here we find a }}$ 50 -fold difference in specificity between ECY2M and ECY2-A1. Evidently our previous preparation of unmodified RNA $^{\mathrm{Tyr}}$ contained a significant proportion of the dimeric conformer, as can be seen for the untreated ECY2 transcript in figure $4 A$ and table $I$. The present observation that the untreated tRNA has kinetic parameters almost identical to those for ECY2D is almost certainly due to the fact that the concentration of the untreated tRNA was calculated assuming that it was entirely in the monomeric form. This would result in an erroncously high $K_{\mathrm{M}}$ and low $V_{\max } / K_{\mathrm{M}}$, in this case coincidently identical to those for ECY2D. The fact that most of the 50 -fold difference between ECY2M and ECY2-A1 is recovered in the dimer, which shares no structural similarities to ECY2M (except for the anticodon arms) suggests that most of the additional binding is of a non-specific nature. It should be noted that ECY2-A1, the microhelix, may also form a dimeric structure This could explain some of the observed difference in activity versus the full length tRNA. We have performed thermal denaturation/renaturation experiments upon ECY2-A1 at relatively low concentrations $(e g 0.5 \mu \mathrm{M})$ and find that the product is indistinguishable from the untreated material (data not shown). We found no evidence for dimerization of the microhelix. Further studies of the microhelix and microhelix analogues are in progress. This report supports our earlier conclusion which postulated that most, if not all of the positive elements for recognition are localized in the anticodon arm.

Gu and Santi have suggesred that the tRNA (m5U54)methyltransferase might recognize and methylate other RNA species due to the fact that oniy the T-arm loop and stem structure is necessary for methylation [17]. These same workers have subsequently found that this enyzme does indeed methylate 16S rRNA in vitio [18]. Our results regarding the tRNA structural elements required for TGT recognition and catalysis suggest that TGT may very well exchange $p r e Q_{1}$ into other RNA species, perhaps leading to queuine incorporation. Further studies are needed to address this possibility.

While the results presented here obtain for the $E$ coli enzyme, they may not hold true for eukaryotic TGTs. Grosjean and coworkers have shown that the entire tRNA molecule appears to be necessary for queuine modification in Xenopus oocytes [19]. This finding, in addition to previous reports that prokaryotic TGTs incorporate a queuine precursor (preQ $\left.Q_{1}\right)$ and do not recognize queuine whoreas the eukaryotic TGTs do recognize queuine, highlights the significant differences between prokaryotic and eukaryotic TGTs with respect to substrate recognition. 


\section{Acknowledgments}

This work was supported by National Institutes of Health Grants GM45968 and GM07767 and the University of Michigan, College of Pharmacy.

\section{References}

I Ditheimer G. Keith G. Sibler AP. Martin RP (1979) Transfer RNA: Structure. Propertics, and Recognition. Cold Spring Harbor Laboratory. Cold Spring Harbor. NY.

2 Crothers DM (1979) Transfer RNA: Strucuure. Properties, and Recognition. Cold Spring Harbor Laboratory. Cold Spring Harbor. NY.

3 Okada N. Nishimura S (1979) Isolation and characterization of a guanine insertion enzyme. a specific IRNA transglycosylase. from Escherichia colli. J Biol Chem 254. 3061-3066

4 Milligan JF. Uhlenbeck OC (1989) Synthesis of small RNAs Using T7 polymerase. Methods En:ymol 180. 51-62

5 Schleich T. Goldstein J (1964) Gel filtration properties of CCD-prepared E coli B SRNA. Proc- Natl Acad Sci USA 52. 744-749

6 Söll D. Cherayil JD. Bock RM (1967) Specificity of IRNA codon recognition as studied by the ribosomal binding technique. J Mol Biol 29, 97-112

7 Zachau HG (1968) Aggregation of serine specific transfer ribonucleic acids. Eur J Biochem 5, 559-566

8 Hampel A. Cherayil J. Bock RM (1971) Specific aggregation of yeast glycine IRNA. Bienelhim Biophurs Acru 228,482-491
9 Loehr JS, Keller EB (1968) Dimers of alanine transfer RNA with acceptor activity. Proc Natl Acad Sci USA 61, 11 i5-1122

10 Yang SK, Söll DG. Crothers DM (1972) Properties of a dimer of tRNATyr (Escherichia coli). Biochemistry 11, 2311-2320

11 Rordorff BF. Kearns DR (1976) Nuclear magnetic resonance investigation of the base-pairing structure of Escherichia coli tRNATyr monomer and dimer conformations. Biochemisury 15, 3320-3330

12 Curnow AW, Kung FL. Koch KA. Garcia GA (1993) tRNA-guanine transglycosylase from Escherichia coli: Gross IRNA structural requirements for recognition. Biochemistry 32. 5239-5246

13 Garcia GA. Koch KA. Chong S (1993) tRNA-guanine transglycosylase from Escherichia cuii: Overexpression. purification, and quaternary structure. J Mol Biol 231, 489-497

14 Grodberg J. Dunn JJ (1988) ompT Encodes the Eicherichia coli outer membrane protease that cleaves T7 RNA polymerase during purificution. J Bacteriel 170, 1245-1253

15 Lubouze E, Bedouelle $H$ (1989) Structural and kinetic basis for the recognitiun of tRNATyr by tyrosyl-tRNA synthetase. J Mol Biol 205.729-735

16 Musier-Forsyth K, Scaringe S, Usman N, Schimmel P (1991) Enzymatic aminoacylation of single-stranded RNA with an RNA cofactor. Proc Natl Acad Sci USA 88, 209-213

17 Gu XG, Santi DV (1991) The T-arn of tRNA is a substrate for the IRNA (m: U54)-methyltransferase. Biochemistry 30, 2999

$18 \mathrm{Gu}$ XG, Ofengand J, Santi DV (1994) In vitro methylation of Escherichia coli 16S rRNA by tRNA (M(5)U54)-methyltransferase. Biochemistry 33. 2255-2261

19 Grosjean H, Edqvist J, Straby K (1993) Dependence of the three-dimensional structure of IRNA for the enzymatic formation of several different IRNA base modifications. Arch Int Physiol Biochim Biophys 101, B13 